\title{
THE QUENCHED CENTRAL LIMIT THEOREM FOR A MODEL OF RANDOM WALK IN RANDOM ENVIRONMENT
}

\author{
VIKTOR BEZBORODOV AND LUCA DI PERSIO
}

\begin{abstract}
In the present paper we provide a proof of the quenched central limit theorem for the random walk in random environment model introduced by Boldrighini, Minlos, and Pellegrinotti in [3].

У цій статті дано доведення квенч-центральної граничної теореми для випадкових блукань у моделі з випадковим середовищем, запропонованій Болдрігіні, Мінлосом і Пеллегринотті [3].
\end{abstract}

\section{INTRODUCTION}

In this article we prove the quenched Central Limit Theorem (CLT) for a model of random walk in random environment, as it has been introduced by Boldrighini, Minlos, and Pellegrinotti, see in particular [3, 4, 6]. At each site the transition probability kernel is affected by the current state of the environment at this site. A more detailed description can be found in Section 2 For a nice overview of the literature on the subject, we refer to [5], and a survey on the recent progress in the related models can be found in [10] or [2]. In [7] the anomalous behavior of the corrections to the CLT in low dimensions has been studied. Moreover, related models are considered in [1] and [9].

We underline that the novelty of this work is that the proofs are essentially based on the the multidimensional martingale CLT by Küchler and Sørensen, see [8].

The paper is organized as follows: in Section 2 we describe the model and give the statement; while in Section 3 we provide all the proofs and some further comments.

\section{Model, COnditions And Results}

Consider a particle moving in a $n$-dimensional infinite lattice and denote by $X_{t}$ is position at time $t$. On the lattice, a dynamical random environment is considered. It is described by the random field

$$
\xi=\left\{\xi_{t}(x): x \in \mathbb{Z}^{n}, t \in \mathbb{Z}^{+}\right\}
$$

Note that the time is discrete. We assume that $\xi$ is the result of independent copies of the same random variable taking values in some finite space $\mathbb{S}$. The space of configurations is given by $\tilde{\Omega}=\mathbb{S}^{\mathbb{Z}^{n} \times \mathbb{Z}^{+}}$. Thus, $\left\{\xi_{t}(x)\right\}_{(x, t) \in \mathbb{Z}^{n} \times \mathbb{Z}^{+}}$is a collection of i.i.d random variables, distributed according to a given probability measure on $\mathbb{S}$ denoted by $\pi$. We denote by $\Pi$ the distribution of $\xi$ in $\tilde{\Omega}$.

The one step transition probability from position $x$ at time $t$ to position $y$ at the subsequent time step $t+1$ is given by

$$
\mathbb{P}\left\{X_{t+1}=y \mid X_{t}=x, \xi\right\}=P\left(y-x, \xi_{t}(x)\right)=P_{0}(y-x)+c\left(y-x, \xi_{t}(x)\right)
$$

where $P_{0}$ is the transition probability of a free random walk and $c$ is the function which provides the influence of the environment on the particle's dynamic. We note that in the original work [3] there was a small factor $\varepsilon$ before the function $c$.

Keywords. Random walk in random environment; quenched central limit theorem; multidimensional martingale central limit theorem. 
In order for the probability $P$ to be well-defined, the following conditions must be fulfilled:

- $0 \leq P(u, s)=P_{0}(u)+c(u, s) \leq 1 \quad \forall s \in \mathbb{S} \quad \forall u \in \mathbb{Z}^{n}$

- $\sum_{u \in \mathbb{Z}^{n}} c(u, s)=0 \quad \forall s \in \mathbb{S}$.

Without loss of generality we assume that the random environment has the following property:

$$
\sum_{s \in \mathbb{S}} c(u, s) \pi(s)=0 \text { for any } u \in \mathbb{Z}^{n},
$$

which means that $P_{0}$ is the mean transition probability. Indeed, if (2.1) is not satisfied, then we can replace $P_{0}(u)$ with $\tilde{P}_{0}(u):=P_{0}(u)+\sum_{s \in \mathbb{S}} c(u, s) \pi(s)$ and $c$ with $\tilde{c}(u, s)=$ $c(u, s)-\tilde{P}_{0}(u)+P_{0}(u)$. This replacement wouldn't change the law of the random walk and (2.1) would hold.

Additionally, let $P_{0}$ and $c$ be of bounded range. We denote by $\mathbb{P}_{\xi}$ the conditional probability with respect to the environment $\xi$.

We will also assume that $\sum_{u \in \mathbb{Z}^{n}} u c\left(u, s_{1}\right)=\sum_{u \in \mathbb{Z}^{n}} u c\left(u, s_{2}\right)$ for $s_{1}, s_{2} \in \mathbb{S}$. It then follows from (2.1) that in fact

$$
\sum_{u \in \mathbb{Z}^{n}} u c(u, s)=0 \quad \forall s \in \mathbb{S} .
$$

Let $Y=\left\{Y_{t}\right\}_{t \in \mathbb{Z}^{+}}$be the stochastic processes defined by $Y_{t}=X_{t}-t b$, where $b=$ $\sum_{u \in \mathbb{Z}^{n}} u P_{0}(u)$. Note that

$$
\sum_{u \in \mathbb{Z}^{n}}(u-b) P_{0}(u)=0 .
$$

For a vector $u \in \mathbb{R}^{n}, u_{i}$ denotes its $i$-th coordinate.

Theorem 1. For almost every realization $\xi$ of the random environment we have

$$
\frac{1}{\sqrt{t}} Y_{t} \Rightarrow \eta^{2} U
$$

$\mathbb{P}_{\xi}$-a.s., where $U$ is a standard normal vector and $\eta^{2}$ is the positive semidefinite matrix with entries

$$
\left(\eta^{2}\right)_{i j}=\sum_{u \in \mathbb{Z}^{n}}\left(u_{i}-b_{i}\right)\left(u_{j}-b_{j}\right) P_{0}(u)
$$

\section{ProOfS}

Lemma 1. For every $\xi \in \tilde{\Omega}$, the process $Y$ is a martingale under $\mathbb{P}_{\xi}$.

Proof. This is a consequence of the definition of $Y$ along with the condition (2.2). Indeed, by (2.3), we have

$$
\begin{gathered}
\mathbb{E}\left[Y_{t+1} \mid Y_{t}, Y_{t-1}, \ldots, Y_{0}\right]=\mathbb{E}\left[Y_{t}+\left(Y_{t+1}-Y_{t}\right) \mid Y_{t}\right] \\
=Y_{t}+\sum_{u \in \mathbb{Z}^{n}}(u-b)\left[P_{0}(u)+c\left(u, \xi_{t}\right)\right]=Y_{t}
\end{gathered}
$$

Let us define the following $n \times n$ matrices: $H_{t}=\mathbb{E}\left(Y_{t} Y_{t}^{\prime}\right)$, where $Y_{t}^{\prime}$ the transposed matrix, the matrix $[Y]_{t}=\left(\left[Y^{i}, Y^{j}\right]_{t}\right)_{1 \leq i, j \leq n}$, and $H_{t}^{\xi}=\mathbb{E}_{\xi}\left(Y_{t} Y_{t}^{\prime}\right)$ (here and below we treat $Y_{t}$ as a column-vector). Let also $\bar{K}_{t}=\frac{1}{\sqrt{t}} I_{n}$, where $I_{n}$ is the $n \times n$ identity matrix. Then, the following result holds true. 
Lemma 2. We have

$$
\begin{gathered}
\mathbb{E}_{\xi}\left[\left(Y_{r+1}^{i}-Y_{r}^{i}\right)\left(Y_{r+1}^{j}-Y_{r}^{j}\right)\right] \\
=\sum_{y \in \mathcal{Y}} \mathbb{P}\left\{Y_{r}=y \mid \xi\right\} \sum_{u \in \mathbb{Z}^{n}}\left(u_{i}-b_{i}\right)\left(u_{j}-b_{j}\right)\left[P_{0}(u)+c\left(u, \xi_{r}(y)\right)\right] .
\end{gathered}
$$

The above sum by $y$ is taken over the countable set

$$
\mathcal{Y}:=\left\{z_{1}+z_{2} b \mid z_{1}, z_{2} \in \mathbb{Z}\right\} .
$$

(Note that $\mathbb{P}\left\{Y_{t} \in \mathcal{Y}\right.$ for all $\left.t \in \mathbb{N}\right\}=1$ ).

Proof. By definition of $Y$ and $\mathbb{P}_{\xi}$, we have

$$
\begin{gathered}
\mathbb{E}_{\xi}\left[\left(Y_{r+1}^{i}-Y_{r}^{i}\right)\left(Y_{r+1}^{j}-Y_{r}^{j}\right)\right] \\
=\mathbb{E}\left[\left(Y_{r+1}^{i}-Y_{r}^{i}\right)\left(Y_{r+1}^{j}-Y_{r}^{j}\right) \mid \xi\right]=\mathbb{E}\left[\mathbb{E}\left\{\left(Y_{r+1}^{i}-Y_{r}^{i}\right)\left(Y_{r+1}^{j}-Y_{r}^{j}\right) \mid \xi, Y_{r}\right\} \mid \xi\right] \\
=\mathbb{E}\left[\sum_{u}\left(u_{i}-b_{i}-Y_{r}^{i}\right)\left(u_{j}-b_{j}-Y_{r}^{j}\right)\left[P_{0}\left(u-Y_{r}\right)+c\left(u-Y_{r}, \xi\left(Y_{r}\right)\right] \mid \xi\right]\right. \\
=\mathbb{E}\left[\sum_{u}\left(u_{i}-b_{i}\right)\left(u_{j}-b_{j}\right)\left[P_{0}(u)+c\left(u, \xi\left(Y_{r}\right)\right] \mid \xi\right]\right. \\
=\sum_{y \in \mathcal{Y}} \mathbb{P}\left\{Y_{r}=y \mid \xi\right\} \sum_{u \in \mathbb{Z}^{n}}\left(u_{i}-b_{i}\right)\left(u_{j}-b_{j}\right)\left[P_{0}(u)+c\left(u, \xi_{r}(y)\right)\right] .
\end{gathered}
$$

Lemma 3. For П-a.a. $\xi$, we have $\mathbb{P}_{\xi}$-a.s.

$$
\frac{\#\left\{r: r \leq t, \xi_{r}\left(Y_{r}\right)=s\right\}}{t} \rightarrow \pi(s), \quad t \rightarrow \infty .
$$

Proof. Recall that $\Pi$ is defined on Page 311. The events $\left\{Y_{r}=y\right\}$ and $\left\{\xi_{r}(y)=s\right\}$ are independent, so (3.7) holds $\mathbb{P}$-a.s. by the law of large numbers. Hence (3.7) also holds

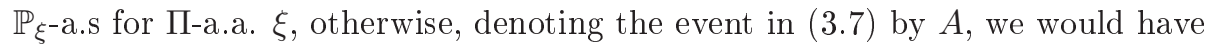

$$
\mathbb{P}(A)=\int \mathbb{P}_{\xi}(A) \Pi(d \xi)<1 .
$$

Lemma 4. We have

$$
\frac{1}{t}[Y]_{t} \rightarrow \eta^{2}
$$

$\mathbb{P}_{\xi}$-a.s. for П-a.a. $\xi$.

Proof. Note that for $1 \leq i, j \leq n$,

$$
\left([Y]_{t}\right)_{i j}=\sum_{0 \leq r<t} \Delta_{r, i j}
$$

where

$$
\Delta_{r, i j}=\left[Y_{r+1}^{i}-Y_{r}^{i}\right]\left[Y_{r+1}^{j}-Y_{r}^{j}\right] .
$$

Under $\mathbb{P}_{\xi}$ conditionally on $\left\{Y_{t}=y\right\}$ the distribution of $Y_{t+1}-Y_{t}$ is $P_{0}(u)+c\left(u, \xi_{t}(y)\right)$. Since under $\mathbb{P}_{\xi}$ the random vectors $Y_{t+1}-Y_{t}$ are independent of each other for different $t$, the statement of the lemma follows from the law of large numbers. Indeed, by (3.7) and the law of large numbers $\mathbb{P}_{\xi}$-a.s.

$$
\sum_{\left\{r: r \leq t, \xi_{r}\left(Y_{r}\right)=s\right\}} \frac{\Delta_{r, i j}}{\#\left\{r: r \leq t, \xi_{r}\left(Y_{r}\right)=s\right\}} \rightarrow \sum_{u \in \mathbb{Z}^{n}}\left(u_{i}-b_{i}\right)\left(u_{j}-b_{j}\right) P(u, s),
$$


and hence by (3.7) we obtain

$$
\sum_{\left\{r: r \leq t, \xi_{r}\left(Y_{r}\right)=s\right\}} \frac{\Delta_{r, i j}}{\pi(s) t} \rightarrow \sum_{u \in \mathbb{Z}^{n}}\left(u_{i}-b_{i}\right)\left(u_{j}-b_{j}\right) P(u, s) .
$$

Therefore, $\mathbb{P}_{\xi \text {-a.s. }}$.

$$
\begin{gathered}
\lim _{t \rightarrow \infty} \sum_{\{r: r \leq t\}} \frac{\Delta_{r, i j}}{t}=\lim _{t \rightarrow \infty} \sum_{s \in \mathbb{S}} \sum_{\left\{r: r \leq t, \xi_{r}\left(Y_{r}\right)=s\right\}} \frac{\Delta_{r, i j}}{t}=\lim _{t \rightarrow \infty} \sum_{s \in \mathbb{S}} \pi(s) \sum_{\left\{r: r \leq t, \xi_{r}\left(Y_{r}\right)=s\right\}} \frac{\Delta_{r, i j}}{\pi(s) t} \\
\sum_{s \in \mathbb{S}} \pi(s) \sum_{u \in \mathbb{Z}^{n}}\left(u_{i}-b_{i}\right)\left(u_{j}-b_{j}\right) P(u, s)=\sum_{u \in \mathbb{Z}^{n}}\left(u_{i}-b_{i}\right)\left(u_{j}-b_{j}\right) P_{0}(u) .
\end{gathered}
$$

Corollary 1. The convergence in Lemma 4 also holds $\mathbb{P}$-a.s.

\section{Lemma 5.}

(i) We have

$$
\left(H_{r+1}\right)_{i j}-\left(H_{r}\right)_{i j}=\sum_{s \in \mathbb{S}} \pi(s) \sum_{u \in \mathbb{Z}^{n}}\left(u_{i}-b_{i}\right)\left(u_{j}-b_{j}\right)\left[P_{0}(u)+c(u, s)\right] .
$$

(ii) We also have

$$
\left.=\sum_{y \in \mathcal{Y}} \mathbb{P}_{\xi}\left\{Y_{r}=y\right\} \sum_{u \in \mathbb{Z}^{n}}\left(H_{r+1}^{\xi}\right)_{i j}-\left(H_{r}^{\xi}\right)_{i j}\right)\left(u_{j}-b_{j}\right)\left[P_{0}(u)+c\left(u, \xi_{r}(y)\right)\right] .
$$

Proof. (i) We start by noting that for $i, j \in\{1, \ldots, n\}$,

Indeed,

$$
\mathbb{E}\left(\left(Y_{t+1}^{i}-Y_{t}^{i}\right) Y_{t}^{j}\right)=0 \text {. }
$$

$$
\begin{gathered}
\mathbb{E}\left(\left(Y_{t+1}^{i}-Y_{t}^{i}\right) Y_{t}^{j}\right)=\mathbb{E} \mathbb{E}\left[\left(Y_{t+1}^{i}-Y_{t}^{i}\right) Y_{t}^{j} \mid Y_{t}\right] \\
=\sum_{y \in \mathcal{Y}} \mathbb{P}\left\{Y_{t}=y\right\} \sum_{u \in \mathbb{Z}^{n}}\left(y_{i}+u_{i}-b_{i}-y_{i}\right) y_{j} P_{0}(u)=\sum_{y \in \mathcal{Y}} \mathbb{P}\left\{Y_{t}=y\right\} y_{j} \sum_{u \in \mathbb{Z}^{n}}\left(u_{i}-b_{i}\right) P_{0}(u)=0
\end{gathered}
$$

by (2.3). Moreover, by (3.12), we have

$$
\begin{gathered}
\left(H_{r+1}\right)_{i j}-\left(H_{r}\right)_{i j}=\mathbb{E}\left(Y_{t+1}^{i} Y_{t+1}^{j}-Y_{t}^{i} Y_{t}^{j}\right) \\
=\mathbb{E}\left(\left(Y_{t+1}^{i}-Y_{t}^{i}\right)\left(Y_{t+1}^{j}-Y_{t}^{j}\right)\right)+\mathbb{E}\left(\left(Y_{t+1}^{i}-Y_{t}^{i}\right) Y_{t}^{j}\right)+\mathbb{E}\left(Y_{t}^{i}\left(Y_{t+1}^{j}-Y_{t}^{j}\right)\right) \\
=\mathbb{E}\left(\left(Y_{t+1}^{i}-Y_{t}^{i}\right)\left(Y_{t+1}^{j}-Y_{t}^{j}\right)\right) .
\end{gathered}
$$

Conditioning on $Y_{t}$, we get

$$
\begin{aligned}
\left(H_{r+1}\right)_{i j}-\left(H_{r+1}\right)_{i j}=\sum_{y} P\left\{Y_{t}=y\right\} \sum_{u}\left(u_{i}-b_{i}\right)\left(u_{j}-b_{j}\right)\left[P_{0}(u)+\mathbb{E}\left[c\left(u, \xi_{r}(y)\right) \mid Y_{t}=y\right]\right] \\
=\sum_{u}\left(u_{i}-b_{i}\right)\left(u_{j}-b_{j}\right) P_{0}(u) .
\end{aligned}
$$

(ii) (3.12) holds for $\mathbb{E}_{\xi}$ too, since

$$
\begin{gathered}
\mathbb{E} \mathbb{E}\left[\left(Y_{t+1}^{i}-Y_{t}^{i}\right) Y_{t}^{j} \mid Y_{t}, \xi\right] \\
=\sum_{y \in \mathcal{Y}} \mathbb{P}\left\{Y_{t}=y\right\} \sum_{u \in \mathbb{Z}^{n}}\left(y_{i}+u_{i}-b_{i}-y_{i}\right) y_{j}\left[P_{0}(u)+c\left(u, \xi_{t}(y)\right)\right]
\end{gathered}
$$




$$
\begin{gathered}
=\sum_{y \in \mathcal{Y}} \mathbb{P}\left\{Y_{t}=y\right\} y_{j} \sum_{u \in \mathbb{Z}^{n}}\left(u_{i}-b_{i}\right) P_{0}(u)+\sum_{y \in \mathcal{Y}} \mathbb{P}\left\{Y_{t}=y\right\} y_{j} \sum_{u \in \mathbb{Z}^{n}} u_{i} c\left(u, \xi_{t}(y)\right) \\
-b_{i} \sum_{y \in \mathcal{Y}} \mathbb{P}\left\{Y_{t}=y\right\} y_{j} \sum_{u \in \mathbb{Z}^{n}} c\left(u, \xi_{t}(y)\right)=0
\end{gathered}
$$

and the proof continues as in $(i)$.

Lemma 6. We have

$$
\frac{1}{t} H_{t} \rightarrow \eta^{2}, \quad \frac{1}{t} H_{t}^{\xi} \rightarrow \eta^{2}
$$

where $\eta^{2}$ is as in (2.5), $\mathbb{P}_{\xi}$-a.s for $\Pi$-a.a. $\xi$.

Proof. Let us only prove the second convergence in (3.13). By Lemma 5 ,

$$
\begin{aligned}
\left(H_{t}^{\xi}\right)_{i j}= & \sum_{r=0}^{t-1} \sum_{y \in \mathcal{Y}} \mathbb{P}_{\xi}\left\{Y_{r}=y\right\} \sum_{u \in \mathbb{Z}^{n}}\left(u_{i}-b_{i}\right)\left(u_{j}-b_{j}\right)\left[P_{0}(u)+c\left(u, \xi_{r}(y)\right)\right] \\
& =t \sum_{u \in \mathbb{Z}^{n}}\left(u_{i}-b_{i}\right)\left(u_{j}-b_{j}\right) P_{0}(u) \\
& +\sum_{u \in \mathbb{Z}^{n}}\left(u_{i}-b_{i}\right)\left(u_{j}-b_{j}\right) \sum_{r=0}^{t-1} \sum_{y \in \mathcal{Y}} \mathbb{P}_{\xi}\left\{Y_{r}=y\right\} c\left(u, \xi_{r}(y)\right) .
\end{aligned}
$$

Since $\sum_{y \in \mathcal{Y}} \mathbb{P}_{\xi}\left\{Y_{r}=y\right\} c\left(u, \xi_{r}(y)\right) \stackrel{(d)}{=} c\left(u, \xi_{r}(\mathbf{0})\right)$ under $\mathbb{P}$ (the symbol $\stackrel{(d)}{=}$ means here 'equal in distribution'), where $\mathbf{0}$ is the origin, and

$$
\sum_{y \in \mathcal{Y}} \mathbb{P}_{\xi}\left\{Y_{r}=y\right\} c\left(u, \xi_{r}(y)\right), \quad r \in \mathbb{N},
$$

constitute a sequence of independent random variables indexed by $r \in \mathbb{N}$, by the law of large numbers for $\Pi$-a.a. $\xi$

$$
\frac{1}{t} \sum_{r=0}^{t-1} \sum_{y \in \mathcal{Y}} \mathbb{P}_{\xi}\left\{Y_{r}=y\right\} c\left(u, \xi_{r}(y)\right) \rightarrow \sum_{s \in \mathbb{S}} \pi(s) c(u, s) .
$$

Combining (3.14) and (3.15) and recalling the definition of $\eta^{2}$, we get the desired result.

Recall that we defined $K_{t}=\frac{1}{\sqrt{t}} I_{n}$.

Proof of Theorem [1] Theorem 2.1 in [8] and Lemmas 4 and 6 imply that $P_{\xi}$-a.s.

$$
\frac{1}{\sqrt{t}} Y_{t} \Rightarrow \eta^{2} U
$$

where $U$ is a standard $n$-dimensional Gaussian vector. The theorem in [8] is formulated for continuous time processes, so to apply it we define $Y_{t}, H_{t}$, etc. for $t \in(1, \infty)$ by $Y_{t}=Y_{\lfloor t\rfloor}, H_{t}=H_{\lfloor t\rfloor}$, etc.

\section{REFERENCES}

[1] G. Barraquand and I. Corwin, Random-walk in beta-distributed random environment, Probability Theory and Related Fields (2016), 1-60.

[2] M. Biskup, Recent progress on the random conductance model, Probab. Surv. 8 (2011), 294-373.

[3] C Boldrighini, R. A. Minlos, and A. Pellegrinotti, Interacting random walk in a dynamical random environment. I. Decay of correlations, Ann. Inst. H. Poincaré Probab. Statist. 30 (1994), no. 4, $519-558$.

[4] C. Boldrighini, R. A. Minlos, and A. Pellegrinotti, Almost-sure central limit theorem for a Markov model of random walk in dynamical random environment, Probab. Theory Related Fields 109 (1997), no. 2, 245-273. 
[5] C. Boldrighini, R. A. Minlos, A. Pellegrinotti, and E. A. Zhizhina, Continuous time random walk in dynamic random environment, Markov Process. Related Fields 21 (2015), no. 4, 971-1004.

[6] K. Boldrigini, R. A. Minlos, and A. Pellegrinotti, Random walks in a random (fluctuating) environment, Uspekhi Mat. Nauk 62 (2007), no. 4(376), 27-76.

[7] L. Di Persio, Anomalous behaviour of the correction to the central limit theorem for a model of random walk in random media, Boll. Unione Mat. Ital. (9) 3 (2010), no. 1, 179-206.

[8] U. Küchler and M. Sørensen, A note on limit theorems for multivariate martingales, Bernoulli 5 (1999), no. 3, 483-493.

[9] T. Thiery and P. Le Doussal, Exact solution for a random walk in a time-dependent $1 d$ random environment: the point-to-point beta polymer, Journal of Physics A: Mathematical and Theoretical 50 (2017), no. 4, 045001.

[10] O. Zeitouni, Random walks in random environments, J. Phys. A 39 (2006), no. 40, R433-R464.

V. BezBorodov: viktor.bezborodov@univr.it

Luca Di Persio: luca.dipersio@univr.it

Dept. of Comp. Science - University of Verona (Italy) 